\title{
JUSTIZ 3.0
}

\section{Christian Gesek, Martin Schneider, Martin Hackl und Thomas Gottwald}

Abstract: Der Einsatz von Technologie verändert die Abläufe in der österreichischen Justiz mittlerweile seit über 40 Jahren. Richtet man den Blick aufdie gesamte Rechtsbranche, so zeigen sich auch klare Parallelen in den damit verbundenen Evolutionsschritten von Legal Tech. Mit der strategischen Initiative Justiz 3.0 arbeitet das BMVRDJ unter enger Einbeziehung der Praxis nun seit 2013 an vollständig digitalen Arbeitsabläufen sowie den dafür notwendigen IT-Arbeitsplätzen. Der Artikel bietet einen Einblick in den Status quo der Digitalisierung in der Justiz, einen Ausblick auf künftige Entwicklungen sowie den dazu korrespondierenden strategischen Überlegungen.

\section{Inhaltsverzeichnis}

A. Einführung und Historie............................................................................................. 222

B. Gemeinsame Prinzipien ...............................................................................................22

C. Aktueller Stand der Arbeiten ..........................................................................................226

D. Der «eCourt» der Zukunft .............................................................................................228

E. Verfahrensmanagement Vorher - Nachher................................................................ 229

F. Ausblick für eine digitale Justiz .................................................................................. 234

G. Die Rolle von künstlicher Intelligenz ……………………………………………... 238

H. Justiz IT und Legal Tech im Gleichschritt................................................................... 239

I. eJustiz-Strategie ........................................................................................................ 241

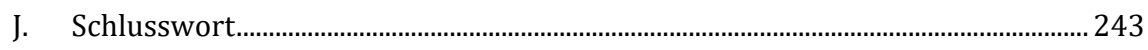




\section{A. EINFÜHRUNG UND HISTORIE}

Die österreichische Justiz-IT blickt mittlerweile auf eine erfolgreiche, bereits mehrere Jahrzehnte umspannende Geschichte zurück. Die Anfänge reichen zurück bis in die 8oer Jahre, als mit der Umstellung des Grundbuchs auf ADV die ersten Schritte unternommen wurden.

Ermutigt durch den Erfolg dieses ersten Projektes wurde die IT-Landschaft im Verlauf der nächsten Jahre und Jahrzehnte um weitere Anwendungen und Services ergänzt.

Es waren dies unter anderem das elektronische Firmenbuch, das elektronische Unterhaltsvorschussverfahren, die Verfahrensautomation Justiz als zentrale Registeranwendung mit zahlreichen Zusatzfunktionen wie automationsunterstützte Gebührenverwaltung und zentrale Zustellung, ebenso die Ediktsdatei als Plattform für Veröffentlichungen oder die Kommunikationsschiene des elektronischen Rechtsverkehrs (ERV).

Bereits in dieser frühen Phase der Automatisierung wurden im Rahmen von universitären Lehrveranstaltungen die Studenten auf die digitale Justiz vorbereitet. Dies war schon deshalb von erheblicher Bedeutung, weil schon bei den frühen Anwendungen nie der Status quo automatisiert wurde, sondern stets auch die Verfahren auf die Möglichkeiten der Informationstechnik hin optimiert wurden, womit zwangsläufig Rechtsänderungen verbunden waren. Damals wurde auch der Satz «Einsatz der Informationstechnik als Hebel zur Erneuerung der Justiz» geprägt.

Auch im Bereich des Strafvollzuges wurde mit den Anwendungen der Integrierten Vollzugs- und Wirtschaftsverwaltungen wertvolle Unterstützung geleistet.

All dies sind Elemente, die heute aus dem Betrieb der Justiz nicht mehr wegzudenken sind und maßgeblich zum reibungslosen Funktionieren beitragen.

Die stetig steigende Schlagzahl technischer und methodischer Innovationen brachte es unweigerlich mit sich, dass viele der Anwendungen funktional und technisch als nicht mehr zeitgemäß und damit erneuerungsbedürftig betrachtet werden mussten.

Verschärfend kam hinzu, dass bereits in den vergangenen Jahren auch in der Justiz ein Anwachsen von digitalen Inhalten zu verzeichnen war, für das jedoch keine adäquate 
Unterstützung etabliert war: gerade im Bereich justizinterner Zusammenarbeit, also bei der Abbildung von Workflow-Prozessen und des Dokumentenmanagements wurde das Korsett der bestehenden Lösungen immer stärker spürbar.

Schlussendlich tat sich gerade in jüngerer Vergangenheit dramatisch beschleunigt eine immer breitere Schere zwischen dem zur Verfügung stehenden Budget und wachsenden Anforderungen auf, die es notwendig machte, am Puls aktueller Entwicklungen weiter zu denken und verbesserte Lösungen zur Verfügung zu stellen.

Vor dem Hintergrund dieser Entwicklungen wurde im Jahr 2013 die strategische Initiative Justiz 3.0 ins Leben gerufen, die kurz gefasst die bestmögliche Optimierung der vorhandenen IT-Unterstützung des Justizbetriebes bis hin zur vollständig digitalen Akten- und Verfahrensführung anstrebt.

In der Phase eins dieser Initiative wurden mehr als 100 Justizmitarbeiter aus allen Bereichen, sowohl Entscheidungsorgane als auch Kanzleimitarbeiter, Rechtspfleger und Gerichtsvollzieher in Arbeitsgruppen einbezogen. In diesen Arbeitsgruppen wurden die wichtigsten funktionalen Geschäftsfelder der Justiz, wie zum Beispiel «Entscheidungen und Verfügungen», «Aktenlauf», aber auch «Eingangs- und Ausgangsprozesse» einer Ist-Betrachtung unterzogen.

Darauf aufbauend wurde - befeuert durch Best-practice-Beispiele aus dem internationalen Umfeld - die Vision eines künftigen «Soll» entwickelt, das heißt ein idealtypisches Bild der künftigen IT-Unterstützung für das jeweilige Geschäftsfeld gezeichnet. Die daraus abgeleiteten Handlungsfelder wurden in Form von Verbesserungspotentialen festgehalten, sodass letztlich zu jedem Geschäftsfeld ein gemeinsames Bild zu den erforderlichen Transformationsmaßnahmen vorlag.

Ebenso beteiligt waren in dieser ersten, etwas länger als ein Jahr dauernden Phase sämtliche hochrangigen Vertreter der Justizverwaltung sowie der Standesvertretungen.

Im Sommer 2014 konnte schließlich ein Endbericht vorgelegt werden, der einerseits das gemeinsame, im nächsten Kapitel skizzierte Verständnis der künftigen Ausrichtung festhielt, andererseits aber auch Grundlage für konkrete Umsetzungsschritte war. In einer ersten Umsetzungsphase dieser Roadmap wurden letztlich die we- 
sentlichen Grundelemente der erforderlichen Querschnittskomponenten entwickelt, sodass im Dezember 2016 der Pilotbetrieb an vier Gerichten gestartet werden konnte.

Das Jahr 2017 war geprägt von einer permanenten Verbesserung von Stabilität und Performance des Gesamtsystems, aber auch vom laufenden Einholen von Feedback und Anregungen der Pilotanwender, die evaluiert und in den Anwendungen umgesetzt wurden. Zusätzlich war aber bereits der weitere Pilotbetrieb in der Gattung Cg am Handelsgericht Wien im Fokus, für den ebenfalls technische und funktionale Erweiterungen notwendig waren.

Im April 2018 konnte plangemäß auch dieser Pilot gestartet werden, aus dem wiederum Erfahrungsgewinn und neue Impulse für die stetige Weiterentwicklung und Verbesserung der Lösungen gewonnen wurden.

Parallel dazu wurden im ersten Halbjahr 2018 die mittel- und langfristigen Ziele einer Digitalisierung justizintern mit den Fachsektionen erarbeitet und schließlich im dritten Quartal in Form eines Vortrages an den Ministerrat festgehalten.

Das Jahr 2019 stand in weiterer Folge im Fokus des Rollouts der digitalen Aktenführung an weiteren Gerichten, wodurch sich die Gesamtanzahl an Gerichten mit digitaler Aktenführung im Zivilverfahren verdoppeln sollte.

Der weitere Ausbau der digitalen Aktenführung auf die nächsten Verfahrensarten und Standorte wird in Abhängigkeit von den verfügbaren Mitteln möglichst zügig vorangetrieben, wobei aktuell an den erforderlichen Adaptierungen für einen Einsatz im Strafverfahren und Justizverwaltung gearbeitet wird. 


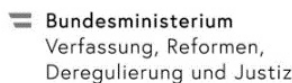

Die Chronologie der Digitalisierungsinitiative Justiz 3.0

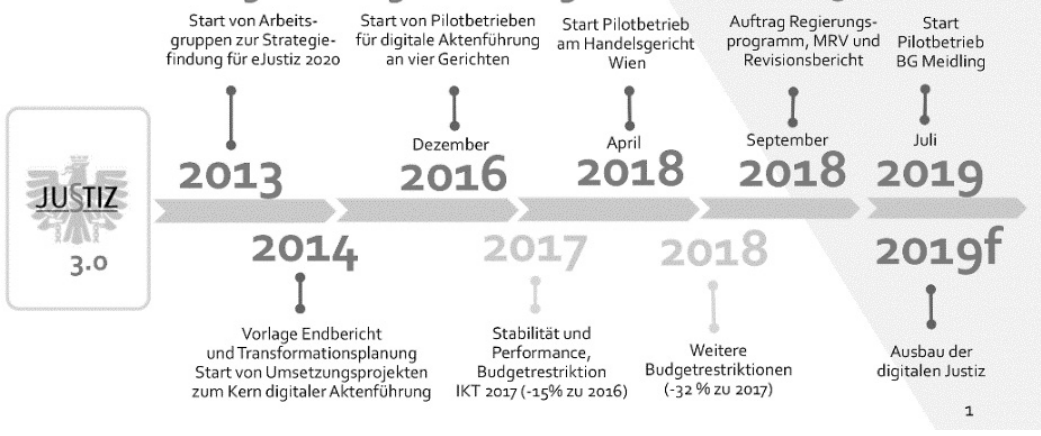

\section{Abbildung 1.}

\section{B. GEMEINSAME PRINZIPIEN}

Als wichtige Eckpfeiler der damit eingeleiteten Digitalen Transformation der Justiz gelten weiterhin die zu Beginn der Initiative gemeinsam definierten Digitalisierungsprinzipien.

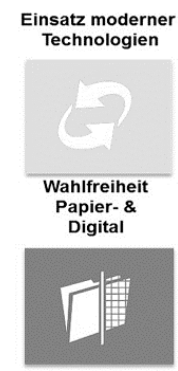

Aufgabenverteilung anhand von Musterprozessen

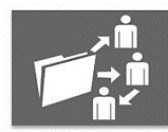

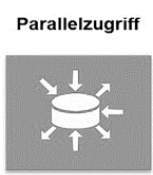

Autonome Arbeitsmöglichkeit

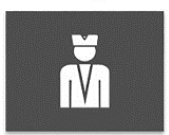

Kommunikation justizintern primär elektronisch

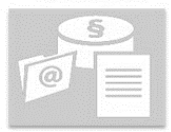

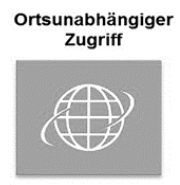

Automatisierter Verfahrensablauf

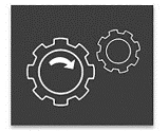

Zustellung von Ausgangsstücken primär zentral und elektronisch

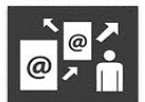

Parallele Aufgabenverteilung
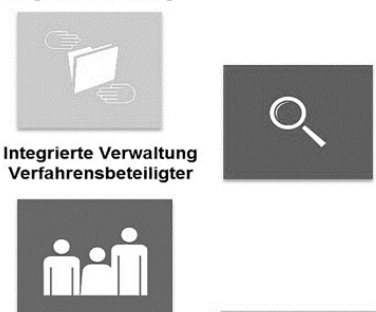

Vollständige Verlaufsinformation zu Gebühren

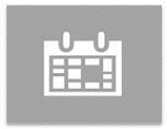

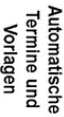

\section{Abbildung 2.}


Zentrales Element ist das Bekenntnis zum vollständig digital geführten Akt in der Justiz:

- Damit verbunden sind die Optionen des ortsunabhängigen Zugriffs auf alle Verfahrensinhalte - ein Zugriff, der auch parallel durch mehrere Berechtigte erfolgen kann - und bereits dadurch großes Potenzial für die künftige Beschleunigung von Verfahren in sich trägt.

- Ein auch im bisherigen Pilotbetrieb bestätigter wesentlicher Vorteil ist in den mit digitalen Inhalten verbundenen Möglichkeiten der Durchsuchbarkeit, Bearbeitung, Weiterverwendung und vielfältiger Strukturierbarkeit des Akteninhaltes zu sehen. Gerade diese Elemente ermöglichen es erst, besonders umfangreiche Akteninhalte effizient zu verwalten.

- Eine weitere Säule stellt der künftig noch verstärkt anzustrebende Ausbau der elektronischen Kommunikation dar, wobei neben der bereits in der Vergangenheit im Fokus stehenden externen Ausrichtung auch der justizinterne Informationsaustausch forciert werden soll.

\section{AKTUELLER STAND DER ARBEITEN}

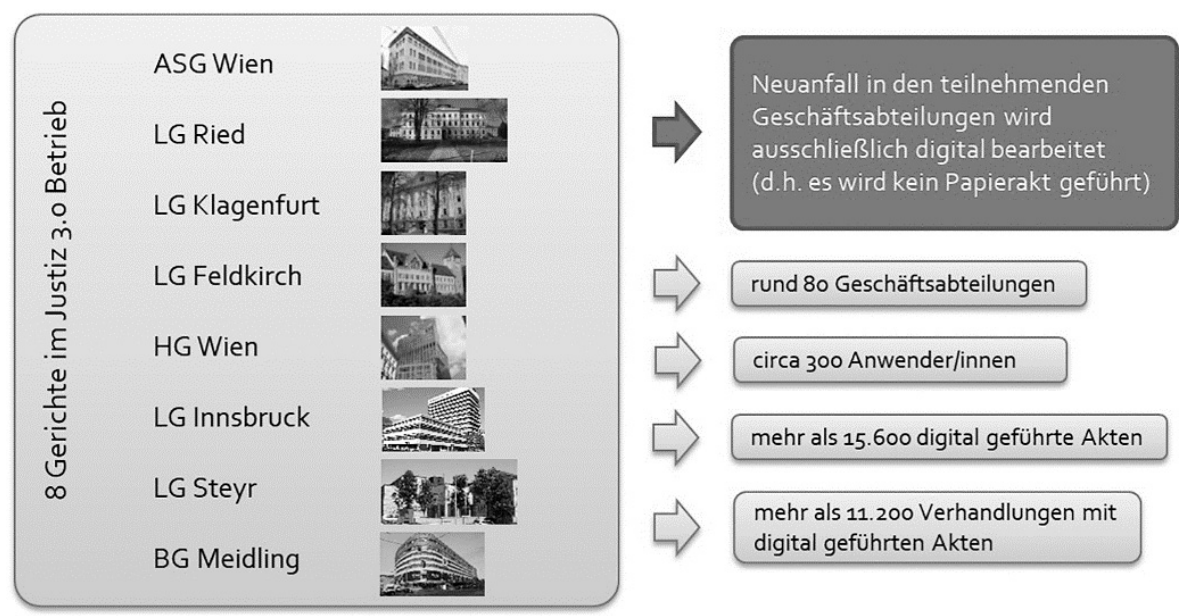

Abbildung 3. 
Mit Stand September 2019 arbeiteten an acht Standorten rund 120 Anwenderinnen und Anwender in Sozial- und Arbeitsrechtsverfahrens, in Handelssachen und in allgemeinen Zivilsachen am Landesgericht und Bezirksgericht. Zum Jahreswechsel 2019/20 waren es bereits zehn Gerichte.

Bislang wurden 15.0oo Akten ausschließlich digital geführt und mehr als 10.0oo Verhandlungen in speziell ausgerüsteten Verhandlungssälen in digital geführten Verfahren abgehalten.

Mit dem Start der Pilotierung am Handelsgericht Wien in streitigen Sachen vor dem Landesgericht wurden die zu bearbeitenden Akten auch schlagartig umfangreicher, womit auch die Vorteile der digitalen Aktenführung noch stärker zum Tragen kommen.
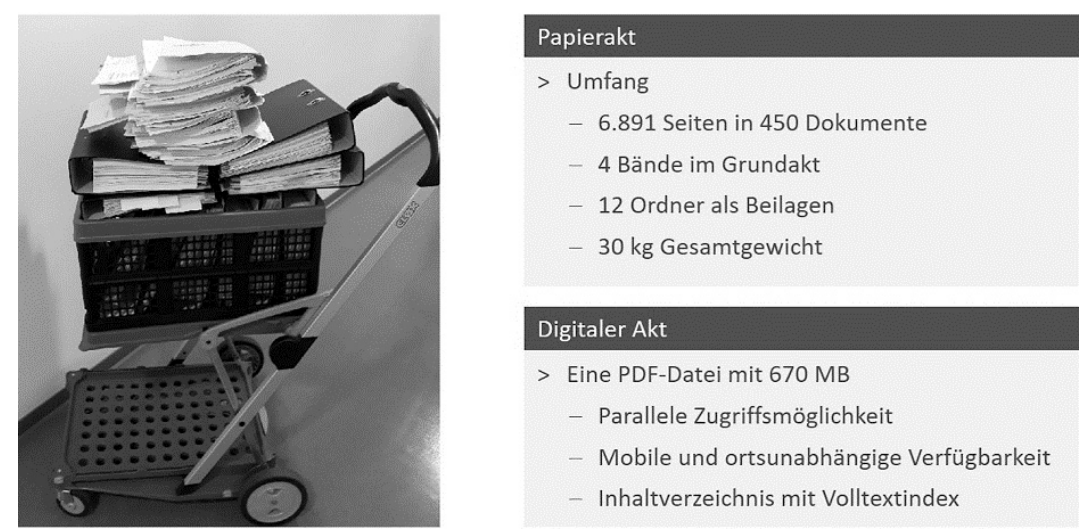

\section{Digitaler Akt}

$>$ Eine PDF-Datei mit $670 \mathrm{MB}$

- Parallele Zugriffsmöglichkeit

- Mobile und ortsunabhängige Verfügbarkeit

- Inhaltverzeichnis mit Volltextindex

Abbildung 4.

Während Justiz 3.o im Bereich der Gerichte aktuell das Ziel der Einführung einer digitalen Aktführung verfolgt, wurden im Strafvollzug einzelne Grundregister umgestellt und unmittelbar auf alle 27 Justizanstalten ausgerollt. Durch geringfügige Anpassung vorhandener Komponenten (wie Aufgabenverwaltung oder Aktensystem) werden künftig auch im Strafvollzug einzelne Verfahren bzw. der Insassen- und Verwaltungsakt als solches vollständig digital abgebildet. 

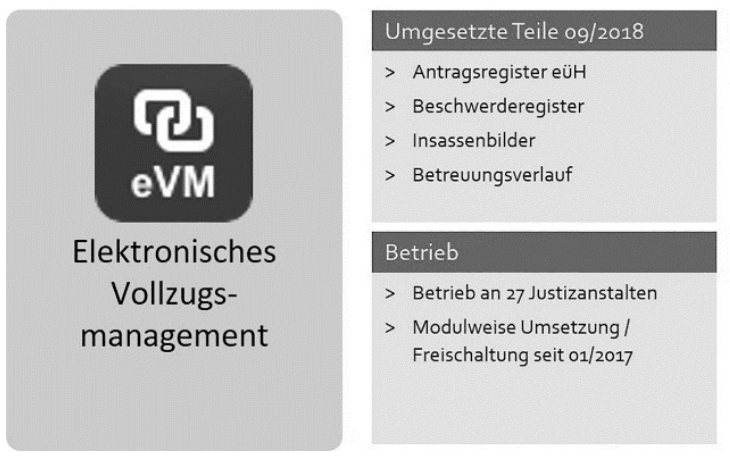

Weitere Schwerpunktsetzung

- Klassifizierung

- Abbildung des Insassenaktes (durch Einsatz JU3० Aktensystem??)

\section{Erneuerung weiterer IVV Module}

- Haftraumverwaltung

- Depositen

- Insassengelderverwaltung

- Vollzugsortsänderung

- Insassenverwaltung

\section{Abbildung 5.}

\section{DER «ECOURT» DER ZUKUNFT}

Eine der zentralen Herausforderungen im Zusammenhang mit der Digitalisierung in der Justiz ist die Ausstattung von Verhandlungssälen, welche vielfältige Anforderungen zu unterstützen hat und andererseits aber auch besonders einfach in der Bedienung sein muss.

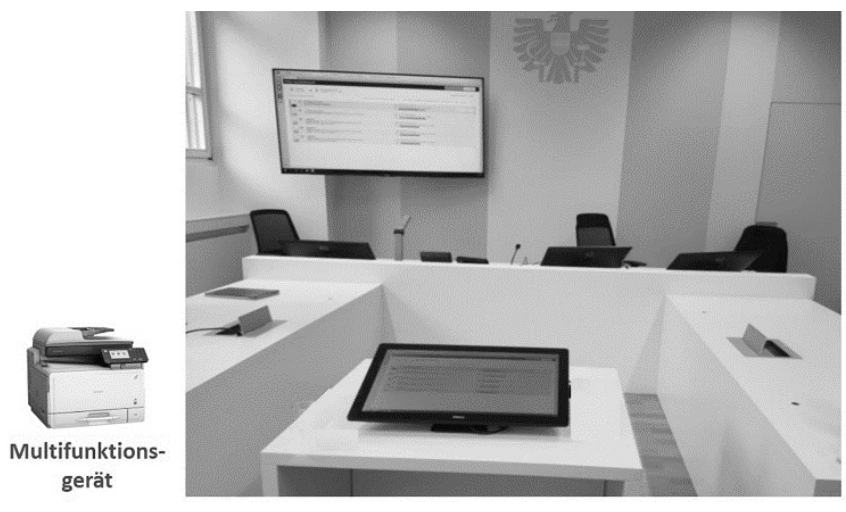

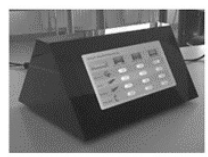

Touch-Panel zur Mediensteuerung

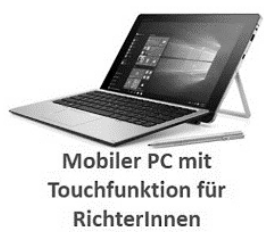

\section{Abbildung 6.}

Wie in seinem Arbeitszimmer findet der Richter im Verhandlungssaal zwei Monitore vor: das Entscheidungsorgan verlässt damit sein Büro, nimmt seinen Tablet-PC mit und 
steckt diesen im Verhandlungssaal über einen Magnetstecker an. Er findet damit automatisch auch im Saal die gewohnte Arbeitsumgebung vor.

An der Rückwand hinter der Richterbank kann ein Großbildschirm angebracht werden, der in den Fällen zum Einsatz kommt, wo der Verhandlungsöffentlichkeit bestimmte Akteninhalte präsentiert werden sollen. Diese Möglichkeit besteht auch vorne am Vernehmungsstand über einen kleineren Monitor, wo dem jeweiligen Verfahrensbeteiligten Akteninhalte gezeigt werden können.

In Abhängigkeit von räumlichen Gegebenheiten und nach laufenden Rückmeldungen aus der Praxis können auch den Parteien und ihren Vertretern Monitore an den jeweiligen Tischen zur Verfügung gestellt werden.

Gesteuert wird diese Infrastruktur über ein Touch-Panel zur Mediensteuerung, sodass mit einer einfachen Matrix Eingangssignal und Ausgangsmedium gewählt werden können. Zusätzlich steht ein Multifunktionsgerät zur Verfügung, das sowohl Scannen als auch Kopieren und Drucken ermöglicht. Damit ist auch für Verfahrenssituationen Vorsorge getroffen, in denen Papier entweder vorgelegt wird oder gedruckt werden soll.

\section{E. VERFAHRENSMANAGEMENT VORHER - NACHHER}
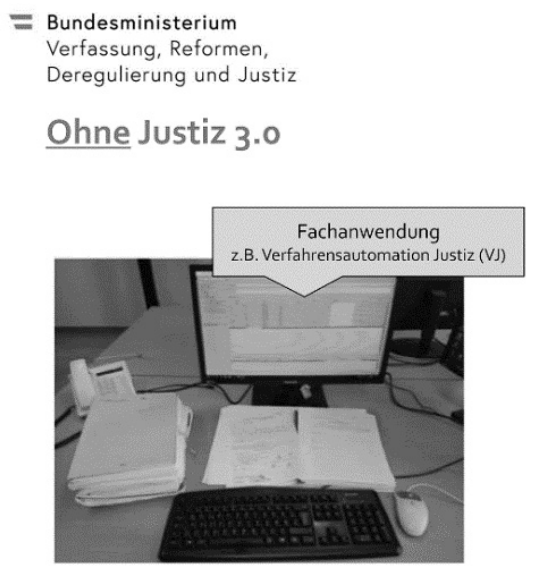
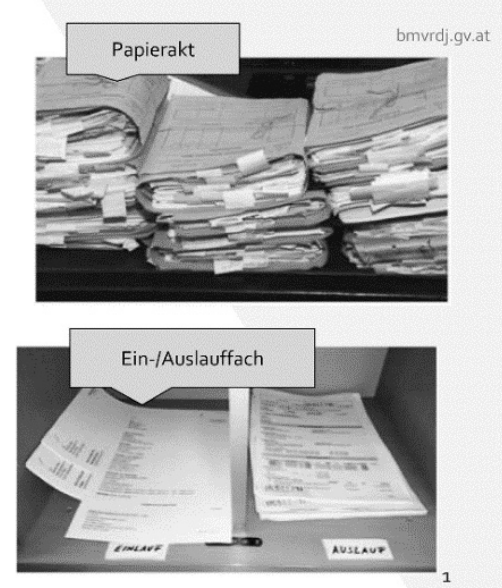

Abbildung 7. 
Im bisherigen Arbeitsumfeld wurde auf einem Bildschirm auf Basis des Papieraktes die Verfahrensautomation Justiz als Registeranwendung aufgerufen, der gesamte Posteingang bzw. Akteneingang und -ausgang war in Papier vorhanden.

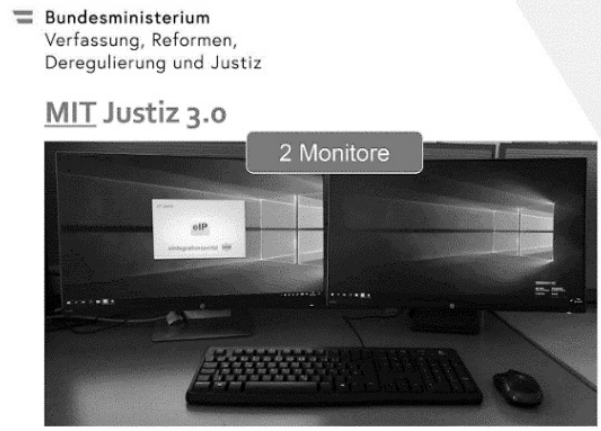

Vorstellung Justiz 3.0 bmvrdj.gvat

$\rightarrow$ Jede Teilnehmerin / jeder

Teilnehmer erhält zwei

Bildschirme die für eine Arbeit im

digitalen Akt erforderlich sind

$\rightarrow$ Justiz 3.0 bedeutet nicht, dass es kein Papier mehr gibt! Einzelne Aktenbestandteile z.B. für das Aktenstudium können gedruckt werden.

$\rightarrow$ Der digitale Akt ist jedoch der führende Akt und ist vollständig zu führen.

\section{Abbildung 8.}

Mit der Digitalisierung stehen jeder Mitarbeiterin und jedem Mitarbeiter zwei Großbildschirme zur Verfügung, wobei diese für Entscheidungsorgane mit Touch-Funktion ausgestattet sind. Der PC wird ersetzt durch einen sogenannten Tablet-PC, der die Vorteile größerer Mobilität und auch ortsunabhängigen Arbeitens mit sich bringt.

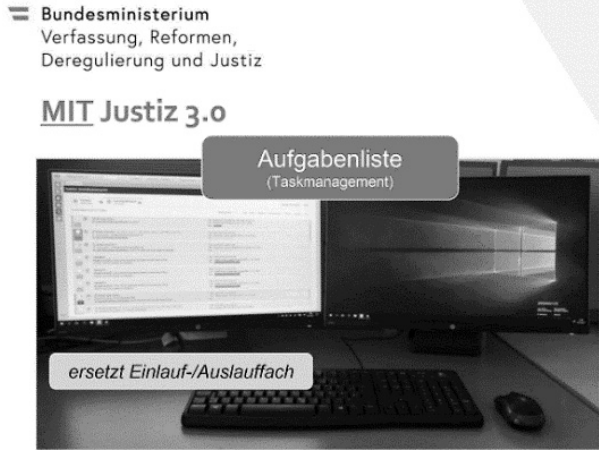

Vorstellung Justiz 3.0 bmvrdj.gv.at

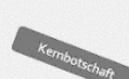

$\rightarrow$ Das Taskmanagement bzw. die Aufgabenliste entspricht einem für die speziellen Bedürfnisse der Justiz angepasstes EmailPostfach.

$\rightarrow$ Aufgaben zu einem Akt werden darin vorgelegt. Ein Eintrag zeigt dabei beispielsweise nicht nur "Absender" und "Betreff", sondern zeigt "Aktenzeichen", eine (kurze) "Rechtsache", das "Dokument" im Anhang (z.B. Klage vom ...) und vieles mehr.

\section{Abbildung 9.}


Auf diesen Bildschirmen wird dann das Task-System, das heißt das digitale Ein- und Auslauffach, aufgerufen.

Diese Aufgabenverwaltung stellt rollen- und organisationsspezifische Sichten auf zu bearbeitende Eingangsstücke und Akten unter Berücksichtigung der jeweils geltenden Geschäftsverteilung zur Verfügung.

Durch Funktionen wie Tagging, Notizen und Stapelbildungen werden verschiedenste Organisationsformen unterstützt, sodass die Arbeit in «großen» und «kleinen» Abteilungen effizient und rasch erfolgen kann.

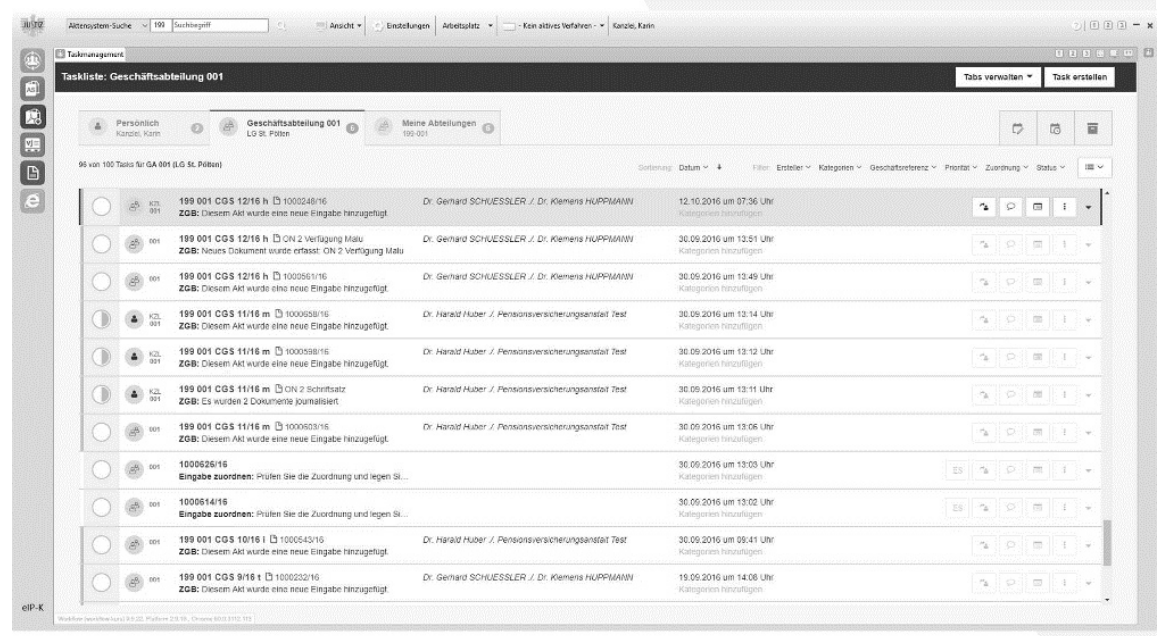

\section{Abbildung 10.}

Nach Anwählen einer Aufgabe öffnet sich - vom Benutzer frei zu definieren - im Fall der Kanzlei zB das Aktensystem, das heißt der Ersatz für den herkömmlichen Papierakt, auf dem zweiten Schirm die Registeranwendung Verfahrensautomation Justiz. 


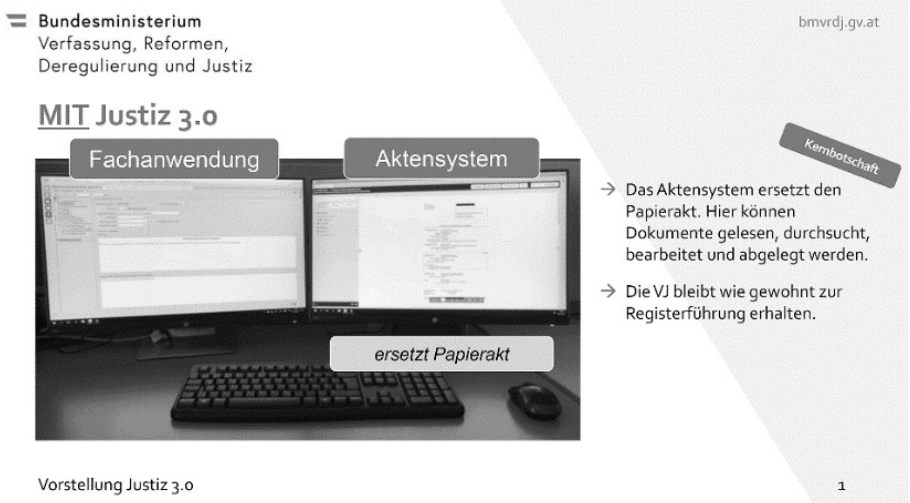

\begin{abstract}
Abbildung 11.
Für das Entscheidungsorgan öffnet sich nach Anklicken der jeweiligen Aufgabe das Aktensystem, das heißt die digitale Abbildung des Akteninhalts. Durch das elektronische Integrationsportal wird sogar eine Verbindung zur jeweils betreffenden Dokumentenstelle im Aktensystem hergestellt, die Grund für die Zuweisung des Akts an die Justizmitarbeiterin oder an den Justizmitarbeiter war.
\end{abstract}

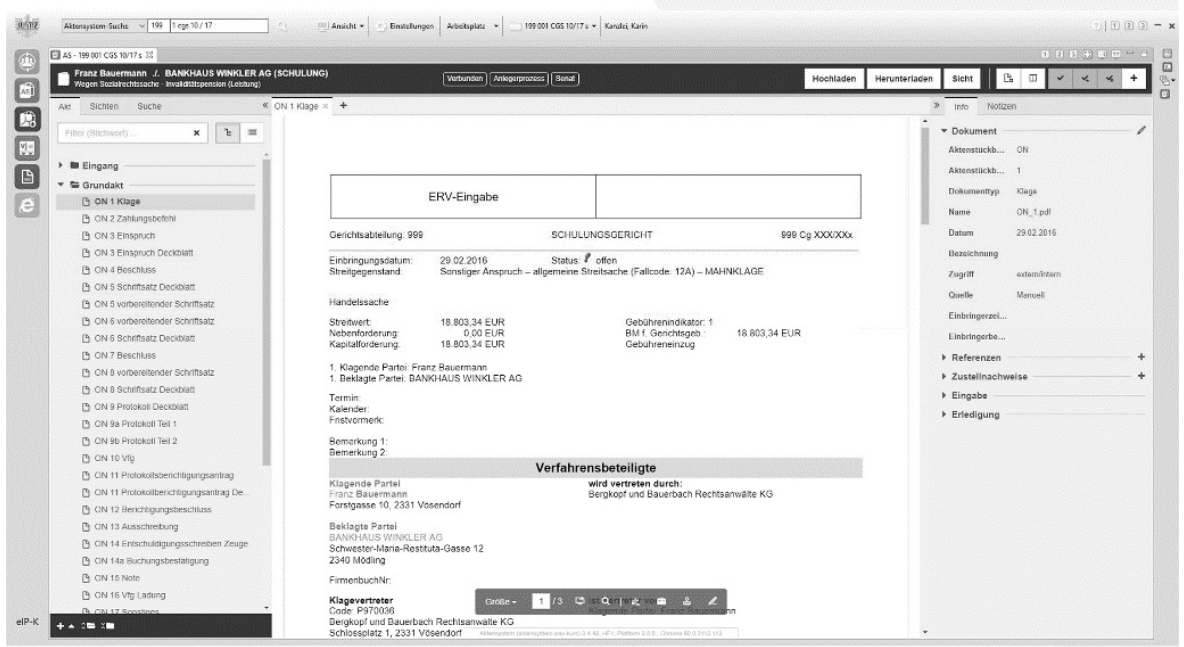

Abbildung 12. 
Im digitalen Akt bestehen dann sämtliche Möglichkeiten der Bearbeitung, Kommentierung sowie des Setzens von Lesezeichen, die auch in der Papierwelt Verwendung finden, aber mit zusätzlicher Funktionalität, so zB der Möglichkeit, diese Anmerkungen für Zwecke der Akteneinsicht wieder auszublenden oder sich auf Basis dieser bereits erfolgten Bearbeitung des Akteninhalts Übersichten, also zielgerichtete Strukturierungen der Dokumente (zB für eine bestimmte Verhandlung oder einen Entscheidungsentwurf) automatisch bilden zu lassen.

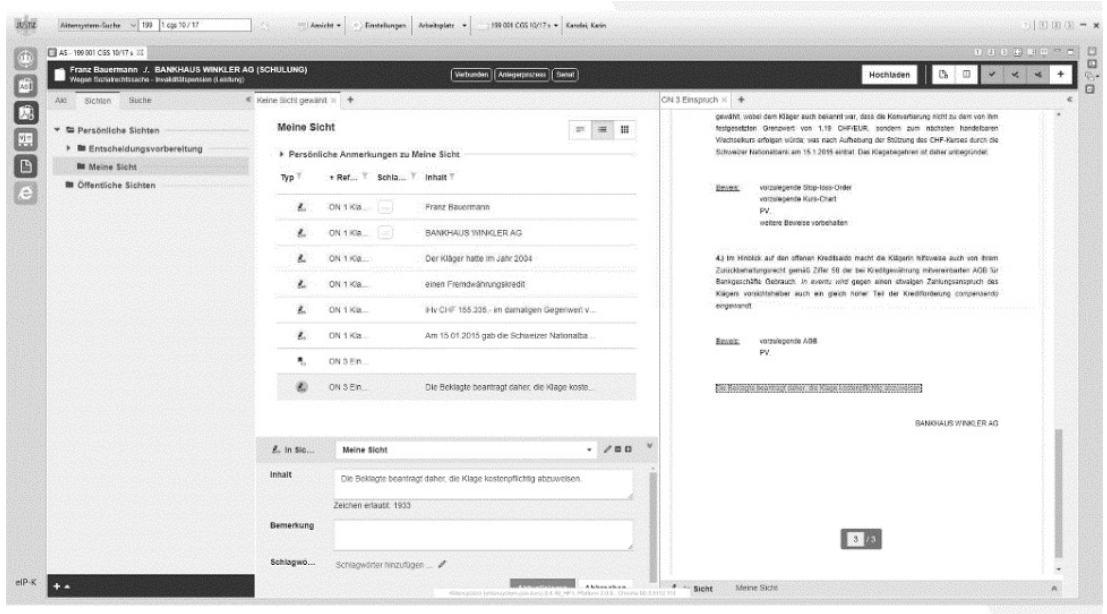

Abbildung 13.

Für das Entscheidungsorgan wird zusätzlich zum digitalen Akt auf dem zweiten Bildschirm beispielsweise die Textverarbeitung für Entscheidungsentwürfe, für Protokolle oder ähnliches geöffnet.

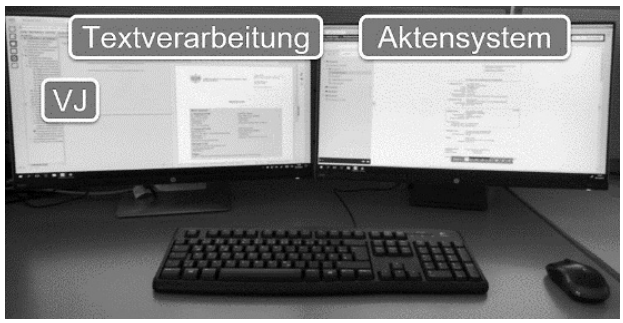

Abbildung 14. 
Als weiterer Vorteil der justizinternen digitalen Aktenführung stehen die digitalen Akten den berechtigten Parteien auch unmittelbar und rund um die Uhr im Wege der elektronischen Akteneinsicht zur Verfügung. Einzelne Akten können selbstverständlich gänzlich oder teilweise (durch Einschränkung einzelner Ordnungsnummern) von der elektronischen Akteneinsicht ausgenommen werden.

\section{Elektr. Akteneinsicht für digitale Akten}

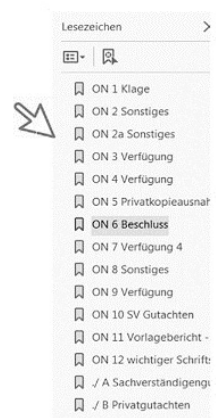

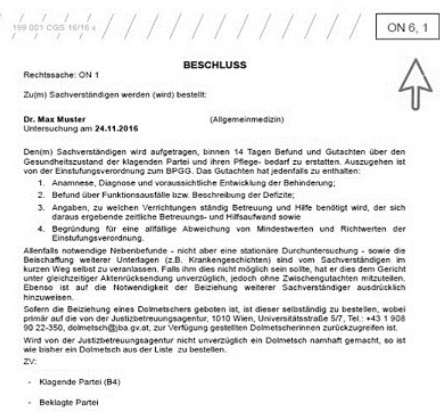

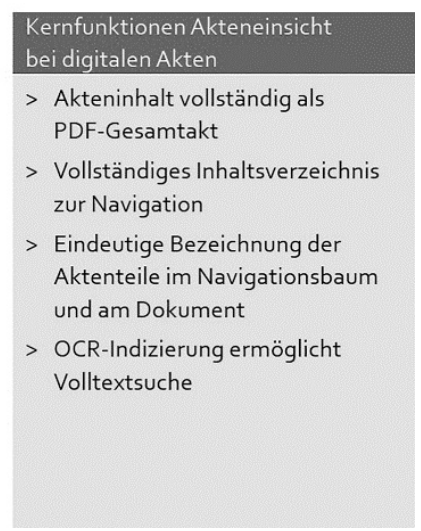

Abbildung 15.

\section{F. AUSBLICK FÜR EINE DIGITALE JUSTIZ}

Da die Digitalisierung eine Vielzahl an Möglichkeiten in unterschiedlichsten Bereichen mit sich bringt, ist der Fokussierung auf wesentliche Ziele sowie einer Schärfung derselbigen besondere Priorität zuzuerkennen, um eine entsprechende Zielverfolgung und -erreichung sicherzustellen. Diese Ziele können anhand mehrere Dimensionen kategorisiert werden, wobei sich eine grundlegende Unterscheidung nach internem und externem Fokus bewährt.

Daran anknüpfend lassen sich aus Sicht der Justiz folgende Wertschöpfungsbereiche ableiten:

- Digitaler Kunde (externer Fokus; Imperativ: «Digitalisiere die Kundenerfahrung»): Einsatz digitaler Technologien für Verfahrensbeteiligte der Justiz (zB Bürger, Rechtsanwälte, Notare, Insassen) 
- Digitale Justiz (interner Fokus; Imperativ «Digitalisiere die Justiz»): Kostensenkung und Prozessoptimierung durch Digitalisierung der internen Prozesse (zB durch Softwareeinsatz)

- Neue digitale Services (Imperativ «Bestehende Services «disrupten»»): Entwicklung neuartiger Services durch Digitalisierung

Gemäß diesen Wertschöpfungsbereichen wurden entsprechende Schwerpunkte der Digitalisierung abgeleitet. Diese können wie folgt eingeordnet werden:

- Digitaler Kunde: Aufbau einer Bürgerservice Plattform mit interaktiven Verfahrensservices

- Digitaler Kunde: Ausbau des elektronischen Rechtsverkehrs (ERV)

- Digitale Justiz: Ausbau des digitalen Verfahrensmanagements durch Justiz 3.0

- Digitale Justiz: Digitalisierung von Verwaltungsverfahren wie dem elektronischem Bildungsmanagement

- Neue digitale Services: Verbesserung der Sicherheit im Strafvollzug durch Detektoren, Sensoren und Videoanalyse

- Neue digitale Services: Einführung digitaler Werkzeuge im Strafverfahren, wie Verhandlungsaufzeichnung sowie Analysewerkzeuge

Versucht man daraus ein Zielbild einer digitalen Justiz zu zeichnen, so lassen sich einige markante Merkmale erkennen - eine Grundvoraussetzung stellt aber in jedem Fall ein digital geführter Akt dar, weil nur damit weitere digitale Funktionen den nötigen Anknüpfungspunkt vorfinden und ihre vollen Vorteile entfalten können.

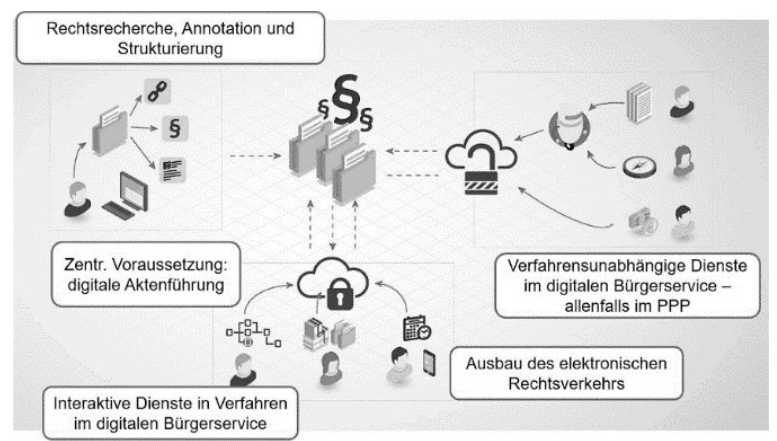

Abbildung 16. 
Im Zivilverfahren sollen daher neben einer flächendeckenden digitalen Aktenführung und einer begleitenden Ausweitung des elektronischen Rechtsverkehrs (ERV) auf weitere Teilnehmerinnen und Teilnehmer verstärkt die Möglichkeiten zur Unterstützung des Entscheidungsorgans durch künstliche Intelligenz genutzt werden. Dies soll durch vorbereitende Rechtsrecherchen, Anbringen von Annotationen auf Dokumenten oder durch vorgeschlagene Aktenstrukturierungen erfolgen. Letztlich ist das Entscheidungsorgan durch Entscheidungsvorschläge (wie bereits im Mahnverfahren vorhanden) zu unterstützen.

Im Bereich des Bürgerservice ist ein strukturierter digitaler Zugangskanal zu erschließen. Über eine interaktive Plattform ist unter Nutzung der e-ID ein Zugang zu «Meinen Verfahren» möglich, womit Dienste wie Auskunft zum aktuellen Verfahrensstand, elektronische Akteneinsicht oder auch Informationen zu Verhandlungsterminen (bzw. auch Push-Informationen bei entsprechenden Verschiebungen) erhalten werden können.

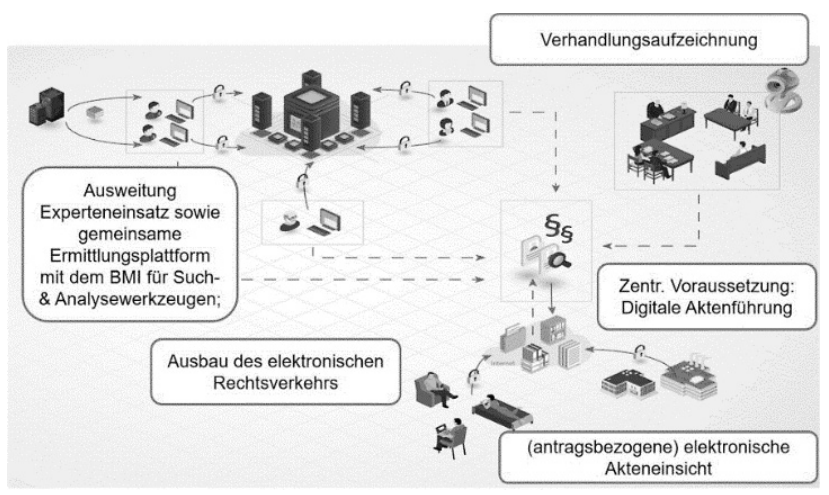

Abbildung 17.

Im Strafverfahren soll der Einsatz von IT-Experten (gerade bei datenbehafteten (Groß)Verfahren) weiter forciert werden. Zur Optimierung der Abläufe zwischen Polizei und Sachverständigen soll eine gemeinsame Ermittlungsplattform geschaffen werden, die den Einsatz von Such- und Analysewerkzeuge ermöglicht.

Durch die Ausweitung der digitalen Aktenführung auf das Strafverfahren werden Abläufe zwischen Gericht und Staatsanwaltschaft vereinfacht und die Möglichkeit für eine 
elektronische Akteneinsicht eröffnet. Im Zuge dessen wird auch der elektronische Rechtsverkehr (ERV) weiter ausgebaut.

Die professionalisierte Aufzeichnung von Hauptverhandlungen im Strafverfahren erlaubt gemeinsam mit intelligenten Markierungen und Transkripten auch ohne schriftliche Protokolle einen raschen und kompakten Überblick zu Sachverhalten.

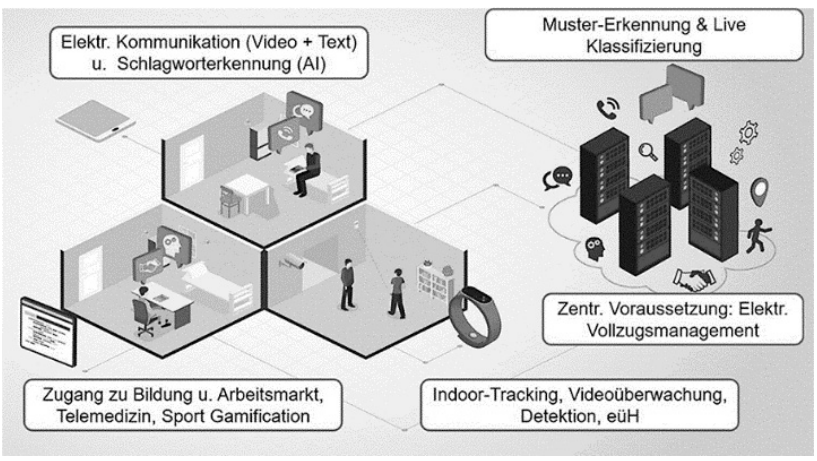

Abbildung 18.

Im Bereich des Strafvollzugs soll durch den Einsatz von IKT die Kommunikation im Rahmen der gesetzlichen Vorgaben automatisiert überwacht und damit die Sicherheit erhöht werden. Durch den Einsatz von Detektoren, Videoüberwachung bzw. auch Indoor-Tracking soll nicht nur der missbräuchliche Einsatz von Smartphones uä verhindert werden, sondern auch die Basis für die Klassifizierung von Insassen anhand von Bewegungsmustern und Kontakten gelegt werden.

Durch die Einbeziehung von Insassen in das digitale Vollzugsmanagement soll das sicherheitstechnische Management von bereits vorhandenen IKT-Geräte bei Insassen verbessert sowie die die nachhaltige Resozialisierung von Insassen gefördert werden. Gamification-Ansätze sollen dabei Anreize für einen adäquaten Umgang, Sport bzw. Bildung schaffen, welche in Kombination mit Tele-Medizin die Betreuungskosten senken sollen.

Durch den flächendeckenden Ausbau des elektronischen Vollzugsmanagements sollen Abläufe transparent und nachvollziehbar gestaltet werden und zu einer Entlastung der Justizwachebeamten von Routinetätigkeiten beitragen. 
Schlussendlich müssen all diese Initiativen und Projekte unter dem Schirm eines koordinierten und abgestimmten Gesamtkonzeptes ihren Platz finden, der e-JustizStrategie.

\title{
G. DIE ROLLE VON KÜNSTLICHER INTELLIGENZ
}

Auch in der aktuellen österreichischen eJustice-Strategie wird «künstliche Intelligenz» (KI; englisch «Artificial Intelligence» (AI)) als die Kerntechnologie der Zukunft für die Justiz angesehen. Um das Schlagwort KI etwas greifbarer zu machen, wird dieser in nachfolgender Grafik in seinen Teilaspekten dargestellt.

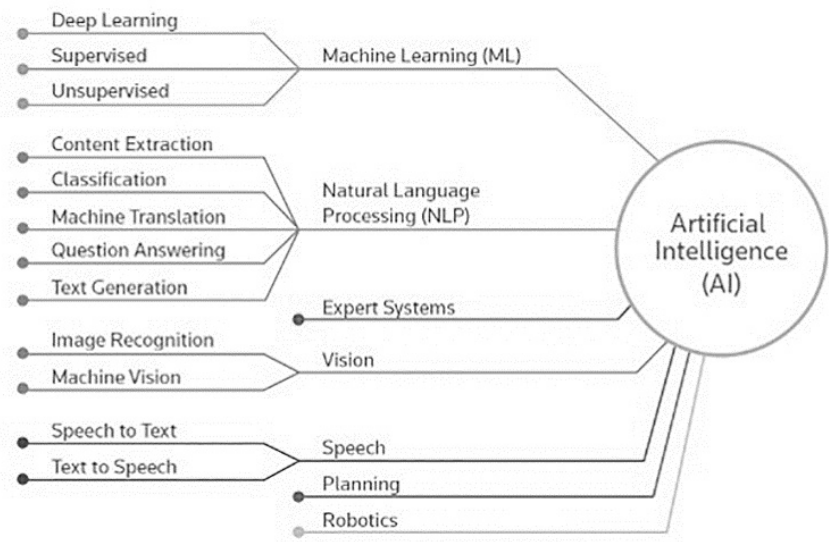

\begin{abstract}
Abbildung 19.
Einige dieser Technologien finden bereits heute Anwendung in unterschiedlichen Justiz-Tools. Beispielsweise werden im Bereich «Knowledge Discovery» mittels «Natural Language Processing» semantische Zusammenhänge aus Texten und Bildern extrahiert werden. Ebenso ist es möglich, Informationen mittels «Machine Learning» zu sortieren, zu klassifizieren und zu filtern. Ein weiteres Anwendungsgebiet ist das Anonymisieren von Textstellen, wie es unter anderem vor der Veröffentlichung von Gerichtsurteilen zu erfolgen hat. Dazu werden die relevanten Textpassagen ebenso mittels «Natural Language Processing» erkannt, klassifiziert und anschließend bei Bedarf anonymisiert werden.
\end{abstract}


Technisch ist dazu seit 2018 ein auf die spezifischen Bedürfnisse der Justiz «trainiertes» KI-Service im Einsatz, welches Schritt für Schritt auf weitere Einsatzbereiche ausgeweitet werden kann. Zum Status quo wird KI in zwei Teilbereichen eingesetzt, wobei hier allen voran Algorithmen aus den Bereichen Machine Learning bzw. Deep Learning zum Einsatz gebracht werden:

- Schritt 1: KI zur Erleichterung der Datenerfassung (Erzeugung von Erfassungsvorschlägen)

- Schritt 2: KI zur Optimierung interner Workflows (Erkennen von Zuständigkeiten)

Die bisher gewonnenen Erfahrungen zeigen dabei das beachtliche Automatisierungspotential durch den Einsatz von KI. In weiterer Folge ist daher ein weiterer Ausbau in komplexeren Aufgabenstellungen naheliegend:

- Schritt 3: KI zur Entscheidungsunterstützung (kontextbezogene Rechtsrecherche)

- Schritt 4: Einsatz von KI zur vollständigen Abbildung ausgewählter Verfahrensschritte

\section{H. JUSTIZ IT UND LEGAL TECH IM GLEICHSCHRITT}

Die «Digitalisierung» ist heutzutage allgegenwärtig - nicht nur im geschäftlichen Umfeld, sondern auch in Freizeit und Privatleben: Besucht man Konzerte und öffentliche Ereignisse, ist man ringsum von Menschen umgeben, welche versuchen, die Momente mit digitalen Mitteln für sich festzuhalten. Digital Austria hat diesen weitreichenden Umstand in folgendem Statement zusammengefasst: «Die Digitalisierung hat die Art, wie wir leben, verändert und sie wird es auch weiterhin tun. In einem Ausmaß, dass wir uns heute noch gar nicht vorstellen können. Digitalisierung betrifft uns alle - alle Lebensbereiche.»

Demzufolge beeinflusst die Digitalisierung auch bereits seit längerer Zeit den Rechtsbereich. Dabei werden diesbezügliche Maßnahmen vielfach unter dem Titel «Legal Tech» zusammengefasst. Damit lohnt sich auch ein näherer Blick auf die Entwicklung von Legal Tech sowie im Vergleich dazu die Entwicklung der Rechtsinformatik in der österreichischen Justiz. Beginnend ab 1980 gab es in der IKT der österreichischen Justiz 
einige wenige monolithische Fachapplikationen, deren Aufgabe klassische Datenverarbeitungen waren. Dass die österreichische Justiz mit dem IKT-Einsatz stets ihrer Zeit voraus war, ist nicht zuletzt daran zu erkennen, dass bereits in dieser frühen Phase der Rechtsinformatik der elektronische Rechtsverkehr (ERV) ins Leben gerufen wurde eine Zielsetzung, welche viele andere Länder in Europa noch vor sich haben. Dieser Zeitraum wird dabei gerne unter dem Titel «Justiz 1.o» eingeordnet und erstreckt sich bis ins Jahr 2000. Nahezu zeitglich dazu werden in der Literatur unter dem Titel «Legal Tech 1.o» Software-Lösungen zur Organisation und Unterstützung von Arbeitsläufen genannt, die hauptsächlich auf Support-Funktionen abzielten.

Die nächste Evolutionsstufe «Justiz 2.0» zwischen 2000 und 2015 machte sich die rasant wachsende Internettechnologie zu Nutze. Wesentliche Verbesserungen waren die Vernetzung der Applikationen sowie eine maßgebliche Automatisierung von Geschäftsprozessen, zB durch die automatische Erstellung von Beschlussentwürfen. Unter dem Titel «Legal Tech 2.0» werden auch hier sehr ähnlich dazu automatisierte Rechtsdienstleistungen verstanden - beispielsweise der Ersatz von Einzelschritten der juristischen Tätigkeit sowie die automatische Erstellung juristischer Dokumente wie Klagen und Beschlüsse.

Ab 2015 wurde mit «Justiz 3.0» der jüngste Evolutionsschritt begangen, dessen Ende aktuell noch nicht absehbar ist. Dabei wird das Ziel einer vollständigen Digitalisierung von Verfahren verfolgt, wozu Benutzerschnittstellen mit besonders hoher User Experience sowie kognitive Technologien zum Einsatz gebracht werden. Passend dazu wird unter dem Titel «Legal Tech 3.0» gar das Bestreben bezeichnet, ein virtuelles und mit künstlicher Intelligenz ausgestattetes Substitut (für Teile von) juristischer Arbeit zu schaffen.

Damit ist unschwer zu erkennen, dass die Entwicklung der gesamten Rechtsbranche starke Parallelen zur Entwicklung des IKT-Einsatzes in der österreichischen Justiz hat - ein Umstand der bei näherer Betrachtung auch mehr als nachvollziehbar ist.

Im Laufe der letzten 40 Jahre wurden in der österreichischen Justiz eine Vielzahl unterschiedlicher Tools entwickelt, womit stets das Ziel verfolgt wurde, den Justizbediensteten die Arbeit zu erleichtern. Im Zentrum stehen dabei natürlich die Fachanwendungen wie beispielsweise: elektronischer Rechtsverkehr (ERV), Elektronisches Grundbuch 
(GB), Verfahrensautomation Justiz (VJ), Elektronische Integrierte Assistenz der Staatsanwaltschaften (EliAS).

Diese werden durch eine Reihe von Assistenzsysteme ergänzt: MOVE - Vorlagen Assistent, Anonymisierungswerkzeuge, Aktenverteilsystem, Normfall Knowledge Discovery für eine Analyse von großen Datenmengen sowie die neu mit Justiz 3.o geschaffenen Systeme eines digitalen Aktensystems und dem Taskmanagement.

«Digitalisierung passiert jetzt - mit oder ohne uns»

Dass die Digitalisierung der Rechtsbranche in vollem Gange ist, zeigt auch ein Blick auf die in diesem Umfeld tätigen Vereine bzw. Institutionen, welche sich maßgeblich zum Ziel gesetzt haben, dass digitale Lösungen, Best Practices und Ideen einer breiten Community zugänglich gemacht werden. Wie bereits erwähnt, wird Digitalisierung zunehmenden Einfluss auf alle Lebensbereiche nach sich ziehen und wird diese mitunter radikal ändern. Erfahrungsgemäß gibt es stets unterschiedliche Herangehensweisen, wie man mit solchen Veränderungen umgeht. Eine besonders treffende Antwort gibt dazu «Burning Man» mit folgendem Statement:

«The appropriate response to new technology is not to angrily retreat into the corner hissing and gnashing your teeth. It's to ask: Okay, how should we use this?»

Und diese Frage, wie man sich diese neuen technologischen Möglichkeiten in der Justiz zu Nutze machen kann, hat sich die österreichische Justiz bereits im Jahr 2013 gestellt und unter dem Titel Justiz 3.o die im Kapitel A beschriebene strategische Initiative ins Leben gerufen.

\section{EJUSTIZ-STRATEGIE}

Die Digitalisierung beschleunigt den Veränderungsprozess der über Jahre hinweg hoch entwickelten IKT-Landschaft der Justiz zunehmend und erfordert Leitlinien und Strategien für eine kontrollierte Transformation. Seit 2006 fasst die für den IKT-Einsatz im Justizressort verantwortliche Abteilung III 3 (Rechtsinformatik und Informations- und Kommunikationstechnologie) die Ziele und Grundsätze dieser Transformation in Form einer IT-Strategie zusammen. Das Gesamtdokument ist unter http://www.justiz.gv.at 
im Bereich «E-Justice» frei abrufbar. Die Kernaussagen lassen sich wie folgt zusammenfassen:

IT-Mission: Die Justiz-IT als zentraler und kompetenter Partner versteht sich als Hebel zur Erneuerung des Justizbetriebs, als moderner und international anerkannter ITDienstleister und setzt die Entwicklung des IT-Einsatzes in der österreichischen Justiz fort, um den IT-Wertbeitrag durch Nutzung innovativer Lösungen und Technologien zu steigern.

IT-Vision: Die Justiz-IT nutzt die Digitalisierung zur Beschleunigung und Vereinfachung von Verfahren sowie zur Bereitstellung zeitgemäßer Services und Zugangskanäle unter Sicherstellung eines optimierten Kosten-Nutzen-Verhältnisses. Dazu stellen fachlich hochqualifizierte, motivierte IT-Mitarbeiterinnen und -Mitarbeiter eine hohe Umsetzungsgeschwindigkeit von Anforderungen bei erforderlicher Qualität und Einhaltung der Vorgaben der Unternehmensarchitektur sicher.

Strategische Zielsetzungen:

- Zeitgemäßes Service für Justiz-Mitarbeiterinnen und -Mitarbeiter, Bürgerinnen und Bürger und berufsmäßige Parteienvertreterinnen und -vertreter.

- Beschleunigung und Vereinfachung von Verfahren durch Digitalisierung

- Erhöhung der Effizienz und Effektivität

- Mitgestaltung von europäischer E-Justiz Strategie und nationalem e-Government

- Plangemäße Umsetzung von IT-Projekten in erforderlicher Qualität

- Sicherheit und weitere qualitative Anforderungen der IT-Lösungen

- Innovativer und kompetenter Partner der Fachbereiche.

- Positives Image der Justiz

Fokusthemen der Zukunft:

- Digitale Transformation

- Künstliche Intelligenz

- Legal Tech

- Management der Unternehmensarchitektur

- Demand- und Projektportfoliomanagement

- Innovationsmanagement 
- Mobilität

- Open Source

- Cloud

IT-Leitlinien:

- Sicherstellen einer autonomen Justiz-IT («Interoperability»)

- Gerichtliche und behördliche Entscheidungen nicht ersetzen, sondern optimal unterstützen («Cognitive assistance»)

- Nutzenmaximierung für die gesamte Justiz («Holisticity»)

- Langfristige Betrachtung bei Zieldefinition und Lösungsentwurf («Sustainability»)

- Einbeziehung neuer Benutzergruppen, ohne bestehende zu benachteiligen («Digital by default»)

- Verfahrensdaten höchstens einmal erfassen sowie Ausbau von Schnittstellen («Once only»)

\section{J. SCHLUSSWORT}

Dem Jubilar Univ.-Prof. DDr. Erich Schweighofer ist zu danken, dass er bei seinen Arbeiten und in der Lehre das Thema «e-Justice» immer im Auge behalten und mitberücksichtigt hat. Dazu hat auch das von ihm mitveranstaltete seit über 20 Jahren stattfindende Internationale Rechtsinformatik Symposium in Salzburg (IRIS) ganz wesentlich beigetragen. Regelmäßig hat der Jubilar mit Nachdruck Beiträge von den Autoren dieses Artikels eingefordert. Mit Fug und Recht kann er schon über viele Jahre als einer der wichtigsten Proponenten der Rechtsinformatik in Österreich genannt werden. 
